\title{
Why Typicality Does Not Explain the Approach to Equilibrium
}

in Mauricio Suárez (ed.): Probabilities, Causes and Propensities in Physics, Synthese Library, Berlin: Springer, 2010, 77-93.

\section{Roman Frigg}

Department of Philosophy, Logic and Scientific Method

London School of Economics

r.p.frigg@lse.ac.uk

www.romanfrigg.org 


\begin{abstract}
Why do systems prepared in a non-equilibrium state approach, and eventually reach, equilibrium? An important contemporary version of the Boltzmannian approach to statistical mechanics answers this question by an appeal to the notion of typicality. The problem with this approach is that it comes in different versions, which are, however, not recognised as such, much less clearly distinguished, and we often find different arguments pursued side by side. The aim of this paper is to disentangle different versions of typicality-based explanations of thermodynamic behaviour and evaluate their respective success. My conclusion will be that the boldest version fails for technical reasons, while more prudent versions leave unanswered essential questions.
\end{abstract}

\title{
Contents
}

1 Introduction 3

2 Classical Boltzmannian SM 4

3 Typicality and the Approach to Equilibrium 6

4 Further Qualms $\quad 15$

5 Conclusion $\quad 21$

$\begin{array}{lr}\text { Acknowledgements } & 22\end{array}$

$\begin{array}{ll}\text { Bibliography } & 22\end{array}$ 


\section{Introduction}

A gas that is confined to the left half of a container uniformly spreads over the entire available space as soon as the confining wall is removed. Yet we never observe the reverse process of a uniformly distributed gas suddenly concentrating in the left half of the container. Such irreversible behaviour is characteristic of many processes and is enshrined in the so-called Second Law of thermodynamics, which, roughly, states that entropy cannot decrease in isolated systems. Statistical mechanics (SM) aims to explain irreversible behaviour in terms of the dynamical laws governing the individual molecules of which the gas is made up. What is it about molecules and their motions that leads them to spread out when the wall is removed? And crucially, what accounts for the fact that the reverse process never happens?

An important answer to these questions was suggested by Boltzmann (1877), and variants of it are currently regarded by many as the most promising option among the innumerable of approaches to statistical mechanics. An important contemporary version of the Boltzmannian approach, originating in the work of Joel Lebowitz (1993a, 1993b), differs from traditional approaches in that it explains irreversibility in terms of the notion of 'typicality'. Intuitively, something is typical if it happens in the 'vast majority' of cases: typical lottery tickets are blanks, typical olympic athletes are well trained, and in a typical series of a thousand coin tosses the ratio of the number of heads and the number of tails is approximately one. The leading idea of a typicality-based approach to SM is to show that thermodynamic behaviour is typical, that is, that the entropy in a system typically increases.

This approach has grown increasingly popular in recent years and has been advocated by a number of authors (references will be given below). The problem with understanding this approach is that it comes in different versions, which are, however, not recognised as such, much less clearly distinguished. We often find different arguments pursued side by side and eventually we end up not having a clear picture of the claims being made. The aim of this paper is to disentangle different versions of typicality-based explanations of thermodynamic behaviour and evaluate their respective success. My somewhat sober conclusion will be that the boldest version fails for technical reasons (having to do with the mathematical structure of the theory), while more prudent versions leave unanswered essential questions.

Before delving into the discussion, two disclaimers are in order. First, this paper only deals with the role typicality plays in explaining the approach to 
equilibrium; what typicality has to offer in response to other problems in $\mathrm{SM}$, in particular to the question of how to reconcile the Gibbsian with the Boltzmannian approach, needs to be discussed elsewhere. Second, typicality has also been invoked in other contexts, for instance in Bohmian mechanics (Dürr et al. 1992; Dürr 2001; Galvan 2006) and in quantum SM (Goldstein et al. 2006). The use of typicality in these theories is beyond the scope of this paper, which is concerned only with classical Boltzmannian SM.

\section{Classical Boltzmannian SM}

Consider a system consisting of $n$ classical particles with three degrees of freedom each. The state of this system is specified by a point $x$, also referred to as the system's microstate, in its $6 n$-dimensional phase space $\Gamma$, which is endowed with the 'standard' Lebesgue measure $\mu_{L} \cdot{ }^{1}$ The dynamics of the system is governed by Hamilton's equations, which define a measure preserving flow $\phi_{t}$ on $\Gamma$, meaning that for all times $t, \phi_{t}: \Gamma \rightarrow \Gamma$ is a one-toone mapping such that $\mu(R)=\mu\left(\phi_{t}(R)\right)$ for all regions $R \subseteq \Gamma$. The system's microstate time $t_{0}$ (its 'initial condition'), $x\left(t_{0}\right)$, evolves into $x(t)=\phi_{t}\left(x\left(t_{0}\right)\right)$ at time $t$. In a Hamiltonian system energy is conserved and hence the motion of the system is confined to the $6 n-1$ dimensional energy hypersurface $\Gamma_{E}$. The measure $\mu_{L}$ can be restricted to $\Gamma_{E}$, which induces a natural invariant measure $\mu$ on $\Gamma_{E}$.

To each macrostate $M_{i}, i=1, \ldots, m$ (where $m$ is finite), of the system, which is characterised by the values of macroscopic parameters such as volume, local pressure and local temperature, there corresponds a set of so-called micro-regions $\Gamma_{M_{i}}$ consisting of all $x \in \Gamma$ for which the macroscopic variables assume the values characteristic for $M_{i}$. The $\Gamma_{M_{i}}$ together form a partition of $\Gamma_{E}$, meaning that they do not overlap and jointly cover $\Gamma: \Gamma_{M_{i}} \cap \Gamma_{M_{j}}=\oslash$ for all $i \neq j$ and $i, j=1, \ldots, m$, and $\Gamma_{M_{1}} \cup \ldots \cup \Gamma_{M_{m}}=\Gamma_{E}$, where ' $\cup$ ', ' $\cap$ ' and ' $\oslash$ ' denote set theoretic union, intersection and the empty set respectively.

The Boltzmann entropy of a macrostate $M_{i}$ is defined as $S_{B}\left(M_{i}\right):=$ $k_{B} \log \left[\mu\left(\Gamma_{M_{i}}\right)\right]$, where $k_{B}$ is the so-called Boltzmann constant. Given this, we define the Boltzmann entropy of a system at time $t, S_{B}(t)$, as the entropy of the system's macrostate at $t: S_{B}(t):=S_{B}\left(M_{x(t)}\right)$, where $x(t)$ is the system's

\footnotetext{
${ }^{1}$ For compact presentations of Boltzmann's account see Goldstein (2001), Goldstein and Lebowitz (2004), Lebowitz (1993a, 1993b, 1999).
} 
microstate at $t$ and $M_{x(t)}$ is the macrostate corresponding to $x(t)$ (i.e. $M_{x(t)}$ is that $M_{i}$ for which it is the case that $x(t) \in \Gamma_{M_{i}}$ at $\left.t\right)$.

The idea now is that the behaviour of $S_{B}(t)$ mirror the behaviour of the thermodynamic entropy $S_{T D}$; that is, it should increase with time $t$ and reach its maximum at equilibrium. Explaining why and how this happens is the central question the Boltzmann approach needs to answer. ${ }^{2}$

Explaining why entropy increases makes sense only if it is far below its equilibrium value to begin with. That this is the case is the subject matter of the so-called past hypothesis, the postulate that the system starts off in a low entropy macro-condition, the 'past state'. Depending on one's stance on reductionism one either takes, with the grand majority of Boltzmannians, the past state to be the Big Bang and the system under investigation to be the entire universe, or, in keeping with the spirit of laboratory physics, one regards states brought about in experimental set-ups (such as the gas being confined to the left half of the container) as the past state and takes the relevant system to be the gas in the box. How this issue is resolved is an important question in its own right, but it is inconsequential for my discussion of typicality. ${ }^{3}$ All that is assumed in what follows is that the system under investigation (whatever it is) be governed by classical Hamiltonian mechanics, isolated from its environment and come into being in a low entropy state. For this reason I adopt a neutral language and from now talk about 'systems', rather than 'the universe', and the 'past state', rather than the 'Big Bang'.

Let $M_{p}$ and $M_{e q}$ be the past and the equilibrium macrostate, and $\Gamma_{M_{p}}$ and $\Gamma_{M_{e q}}$ the respective micro regions (for ease of notation later on I assume, without loss of generality, that macrostates are labelled such that $M_{p}=M_{1}$ and $M_{e q}=M_{m}$ ). The explanandum then is this: given that the system's macrostate at $t_{0}$ is $M_{p}$ (i.e. given that the system's microstate $x\left(t_{0}\right)$ lies within $\Gamma_{M_{p}}$ at $t_{0}$ ), why does the Boltzmann entropy increase as time unfolds and why does the system eventually reach equilibrium (i.e. why does the system's microstate $x(t)$ eventually wind up in $\left.\Gamma_{M_{e q}}\right)$ ?

The standard Boltzmannian response is to introduce a probability measure over the $M_{i}$ and to argue that these probabilities come out such that

\footnotetext{
${ }^{2}$ This 'mirroring' need not be perfect and occasional deviations of the Boltzmann entropy from its thermodynamic counterpart are no cause for concern (Callender 1999; 2001).

${ }^{3}$ If one takes the past state to be the state at the beginning of the universe, there is the further question of whether or not one needs to explain why the world came into being in such a special state. For opposite views on that matter see the contributions of Callender and Price to Hitchcock (2004).
} 
the system is, in one way or another, overwhelmingly likely to evolve in such a way that entropy increases and it eventually reaches $M_{e q}$ (see Frigg (2008, Sec. 2) for a discussion of this approach). The main problem with this response is that at some point it inevitably has to invoke ergodicity, a notion which is notoriously beset with problems (Earman \& Rédei 1996). Typicality approaches promise to eschew such commitments and provide an explanation of the approach to equilibrium free of unmanageable notions like ergodicity.

\section{Typicality and the Approach to Equilib- rium}

Consider an element $e$ of a set $\Sigma$. Typicality is a relational property of $e$, which $e$ posses with respect to $\Sigma$, a property $P$ and a measure $\nu$, often referred to as 'tyicality measure'. ${ }^{4}$ Roughly speaking, $e$ is typical if most members of $\Sigma$ have property $P$ and $e$ is one of them. More precisely, let $\Pi$ be the subset of $\Sigma$ consisting of all elements that have property $P$. Then the element $e$ is typical iff $e \in \Pi$ and $\nu_{\Sigma}(\Pi):=\nu(\Pi) / \nu(\Sigma) \geq 1-\varepsilon$, where $\varepsilon$ is a finite but small positive real number; $\nu_{\Sigma}(\cdot)$ is referred to as the 'measure conditional on $\Sigma$ ', or simply 'conditional measure'. ${ }^{5}$ Derivatively, one can then refer to $\Pi$ as the 'typical set (with respect to $\Sigma$ and $\nu$ )' and to those elements that possess property $P$ (i.e. the members of $\Pi$ ) as 'typical elements (with respect to $\Sigma, P$, and $\nu$ )'. Conversely, an element $e$ is atypical iff it belongs to the complement of $\Pi, \Omega:=\Sigma \backslash \Pi$, in which case we refer to $\Omega$ as the 'atypical set' and to its members as 'atypical elements'. For instance the number $\pi$ is typical with respect to the interval $[0,1]$, the property 'not being specifiable by a finite number of digits' and the usual Lebesgue measure on the real numbers because it is a theorem of number theory that the set of all

\footnotetext{
${ }^{4}$ Tyicality measures often are, but need not be, probability measures (Zanghì 2005, 188).

${ }^{5}$ This definition of typicality is adapted from Dürr (1998, Sec. 2), Lavis (2005, 258), Zanghì $(2005,185)$, and Volchan $(2007,805)$. Strictly speaking one should refer to this notion as ' $\varepsilon$-typicality' because the definition depends on the choice of $\varepsilon$ and elements that are typical with respect to one choice of $\varepsilon$ need not be typical with respect to another. However, nothing in what follows depends on a particular choice of $\varepsilon$ and so there is no need to make this dependence explicit. Furthermore, there is an alternative definition of typicality which is stricter than the one adopted here in that it requires $\nu(\Pi) / \nu(\Sigma)=1$. This definition is unsuitable in the present context because it classifies as atypical certain elements that, from a physics point of view, clearly are typical.
} 
numbers that have this property has measure one. Similarly, numbers in the interval $[1 / 2,1 / 2+\varepsilon / 2]$ are atypical in $[0,1]$ because $[0,1] \backslash[1 / 2,1 / 2+\varepsilon / 2]$ has Lebesgue measure greater than $1-\varepsilon$.

The element of interest in SM is a microstate $x$. Different approaches to SM disagree about the choice of the set $\Sigma$ and about the selection of a relevant property $P$; they all agree that the typicality measure is the Lebesgue measure $\mu$ (I discuss this assumption in the next section). In this section I show that typicality is used in (at least) three different ways to explain why a system like a gas approaches equilibrium and argue that none of them is successful.

Before discussing these approaches an important technical result needs to be stated. Under certain circumstances (I come back to these in Section 4) it is the case that $\Gamma_{M_{e q}}$ is the largest of all $\Gamma_{M_{i}}$ (relative to the Lebesgue measure $\mu$ ); in fact, for large $n$ it is vastly larger than the area of all other regions (Ehrenfest \& Ehrenfest 1912, 30). Numerical considerations show that the ratio $\mu\left(\Gamma_{M_{e q}}\right) / \mu\left(\Gamma_{M_{i}}\right)$, where $M_{i}$ is a 'standard' non-equilibrium macrostate (e.g. one of the kind in which the gas is confined to the left half of the container), is of the magnitude of $10^{n}$ (Goldstein 2001, 43; Penrose $1989,403)$. For want of a better term I refer to this matter of fact as the 'dominance of the equilibrium macrostate'.

This dominance is then often glossed as implying (or being equivalent to the fact) that for large $n, \Gamma_{E}$ is almost entirely taken up by equilibrium microstates; in other words, it is glossed as the fact that equilibrium microstates are typical with respect to $\Gamma_{E}$ and the Lebesgue measure $\mu$ (Bricmont 1995, 146; Goldstein 2001, 43; Zanghì 2005, 191, 196). As we shall see in Section 4, gloss is not generally true. However, for the sake of argument I assume throughout this section that we are dealing only with systems for which this gloss is correct.

Account 1. A first account of why systems behave thermodynamically is suggested by Goldstein (2001) and explains this fact in terms of the dominance of the equilibrium macrostate:

' $\left[\Gamma_{E}\right]$ consists almost entirely of phase points in the equilibrium macrostate $\left[\Gamma_{M_{e q}}\right]$, with ridiculously few exceptions whose totality has volume of order $10^{-10^{20}}$ relative to that of $\left[\Gamma_{E}\right]$. For a non-equilibrium phase point $[x]$ of energy $E$, the Hamiltonian dynamics governing the motion $[x(t)]$ would have to be ridiculously special to avoid reasonably quickly carrying $[x(t)]$ into $\left[\Gamma_{M_{e q}}\right]$ and 
keeping it there for an extremely long time - unless, of course, $[x]$ itself were ridiculously special.' (Goldstein 2001, 43-44) ${ }^{6}$

Some pages further down he summarises his view as follows:

'Suppose a system, e.g. a gas in a box, is in a state of low entropy at some time. Why should its entropy tend to be larger at a later time? The reason is basically that states of large entropy correspond to regions in phase space of enormously greater volume than those of lower entropy.' (Goldstein 2001, 49).

These passages allow for two readings. On the first - and more obvious reading, Goldstein suggests that a system approaches equilibrium simply because the overwhelming majority of states in $\Gamma_{E}$ are equilibrium microstates; in other words, it approaches equilibrium simply because equilibrium microstates are typical and non-equilibrium microstates are atypical (with respect to $\Gamma_{E}$ and $\mu$ ). This also seems to be Zanghì's view when he writes that

'reaching the equilibrium distribution in the course of the temporal evolution of a system is inevitable due to the fact that the overwhelming majority of microstates in the phase space have this distribution; a fact often not understood by the critics of Boltzmann [...]' (Zanghì 2005, 196; my translation)

This point of view contrasts with one that explains the approach to equilibrium by appeal to specific dynamical properties such as ergodicity or mixing. Goldstein dismisses the view that either of these properties could play any role in the foundation of SM as 'thoroughly misguided' $(2001,45):^{7}$

'Boltzmann's key insight was that, given the energy of a system, the overwhelming majority of its phase points on the corresponding energy surface are equilibrium points, all of which look macroscopically more or less the same. This means that the value of any

\footnotetext{
${ }^{6}$ Square brackets indicate that Goldstein's notation has been replaced by the notion used in this paper. I will use this convention throughout.

${ }^{7}$ Albert takes a similar stance and dismisses approaches to the foundations of SM that appeal to ergodicity as 'sheer madness' $(2000,70)$ and ergodic theory as an enterprise that has 'produced beautiful mathematics' but is ultimately, if we are interested in the foundation of SM, 'nothing more nor less [...] than a waste of time' (ibid.).
} 
thermodynamic quantity is, to all intents and purposes, constant on the energy surface, and averaging over the energy surface will thus reproduce that constant value, regardless of whether or not the system is ergodic.' (Goldstein 2001, 45)

This criticism is not specific to ergodicity and could just as well be levelled against any other dynamical property that a system could posses. This suggests that dynamical considerations are regarded as irrelevant for an explanation of the approach to equilibrium and a system eventually reaches equilibrium just because equilibrium conditions are typical.

This is not so. In general there is no reason to assume that points in an atypical set have to evolve into a typical set; typical states do not per se 'attract' atypical states. Uffink (2007, 979-980) provides the following example. Consider a trajectory $x(t)$, i.e. the set $\left\{x(t)=\phi_{t}\left(x\left(t_{0}\right)\right) \mid t \in\left[t_{0}, \infty\right)\right\}$, a set of measure zero in $\Gamma_{E}$. Its complement, the set $\Gamma_{E} \backslash x(t)$ of points not laying on $x(t)$, has measure one. Hence the points on $x(t)$ are atypical while the ones not on $x(t)$ are typical (with respect to $\Gamma_{E}, \mu$, and the property 'being on $\left.x(t)^{\prime}\right)$. But from this we cannot conclude that a point on $x(t)$ eventually has to move away from $x(t)$ and end up in $\Gamma \backslash x(t)$; in fact the uniqueness theorem for solutions tells us that it does not (for a discussion of uniqueness theorems see Arnold (2006)). The moral is that non-equilibrium states do not evolve into equilibrium states simply because there are overwhelmingly more of the latter than of the former, i.e. because the former are atypical and the latter are typical. It does not somehow lie in the 'nature' of atypical states to evolve into typical ones.

One might reply that this example does not fit the mould because the claim is not that any typical set is such that trajectories having atypical initial conditions eventually wind up in the typical set; the claim rather is that this is a special feature of the set that is typical with respect to the property of being an equilibrium state.

But why should this be so? Equilibrium is defined solely in terms of macroscopic quantities and without any reference to the system's dynamics. Why, then, should it be the case that the micro-dynamics is such that it carries atypical points into the typical set? The fact that the there are many more typical than atypical points does not in any way imply that the latter have to evolve towards the former. In other words, if a system is in an atypical microstate (which it is by the Past Hypothesis), it does not evolve into an equilibrium microstate just because the latter are typical. Whether 
or not this happens depends on the dynamics of the system, and whether the dynamics is of the right kind is a question that cannot be answered by appeal to measure-theoretic arguments about the system's macrostate structure.

Account 2. If a given non-equilibrium microstate eventually evolves into an equilibrium microstate this happens due to the dynamics of the system, which is determined by equations of motion and the system's Hamiltonian. Hence an account that disregards dynamical consideration and tries to explain the approach to equilibrium solely by appeal to considerations having to do with the measures of macrostates is doomed to failure. So the question remains: what dynamical conditions does the system have to satisfy for it to approach equilibrium? On the second reading of the first of the above quotations, Goldstein offers at least the beginning of an answer to exactly this question when he restricts his claim that systems reach equilibrium quickly to a dynamics that is not 'ridiculously special' and to initial conditions that are not 'ridiculously special' either. This clearly is a condition on the dynamics of the system, albeit not a very informative one because Goldstein does not tell us what he means by 'ridiculously special'. The only indication of what non-ridiculously-specialness could consist in is contained in the following remark:

'The dynamics of the system prefers a given equilibrium point neither more nor less than it prefers any other given phase point, even a specific far-from equilibrium phase point, corresponding say to the leftmost snapshot.' (Goldstein 2001, 42)

Stripped of its anthropomorphisms, this passage might be read as saying that sooner or later $x(t)$ visits every point in $\Gamma_{E}$, which is just Boltzmann's original definition of ergodicity (see Sklar 1993, 160). However, as is well known, there are no trajectories that satisfy this condition (in phase spaces of more than one dimension). An obvious way to fix the problem would be to substitute the modern definition of ergodicity (roughly that the system's state visits every subset of finite measure at some point and spends an amount of time in it that is proportional to the subset's volume) for Boltzmann's. However, given Goldstein's polemic against ergodicity this can hardly be the dynamical condition that he envisages.

So the crucial question is still unanswered: what are the properties of the dynamics of a system that exhibits the right kind of entropy increasing behaviour? Surprisingly, this question has hardly attracted any attention so 
far; in fact, I am aware of only two proposed answers. The first is due to Bricmont, who tentatively puts forward the suggestion

'that some form of mixing is important for the approach to equilibrium to take place (after all, for the harmonic oscillator we have neither approach to equilibrium nor any form of mixing), but only in some kind of reduced phase space $\left(\mathbf{R}^{2}\right.$ here [i.e. in the example of a system of $N$ uncoupled anharmonic oscillators of identical mass]), determined by the macroscopic variables.' (Bricmont 2001, 16)

Bricmont himself is clear that this is only a 'suggestion' that he does 'not know how to formulate precisely' (ibid.), and that it is still an open question whether, and if so how, this suggestion can be generalised to yield a general condition that would do the work that ergodicity (with respect to the entire phase space) was supposed to in the orthodox approach to SM.

The second suggestion departs from Lavis' (2008, Sec. 2) observation that the Kac ring model, which, as is well known, behaves theormodynamically while failing to be ergodic (see also Bricmont 2001, 10-14), in fact has an ergodic decomposition. This suggests that having such a decomposition plays a part in explaining the approach to equilibrium. Again, the difficulty is that this observation is made in the context of a particular example and it is not at present clear whether, and if so how, it could be generalised to yield a general necessary condition for the approach to equilibrium to take place.

These two suggestions point in the right direction. The question is whether they can be given a precise and general formulation, and whether it is possible to show that realistic systems actually obey one of them. A further question concerns the relation between these (and potential other) conditions. Is one a special case of the other? If not, do they belong to a family of conditions that have certain important features in common? These are important questions that should be addressed in the future.

Account 3. An altogether different line of argument can be found in Lebowitz (1993a, 1993b, 1999) and Lebowitz \& Goldstein (2004) and (possibly) Zanghì (2005, Sec. 2.4.4) The difference lies in the fact that what I refer to as Account 3 focusses on the internal structure of the micro-regions $\Gamma_{M_{i}}$ rather than the entire phase space. The core of this view is captured in the following quotation: 
"By "typicality" we mean that for any $\left[\Gamma_{M_{i}}\right][\ldots]$ the relative volume of the set of microstates $[x]$ in $\left[\Gamma_{M_{i}}\right]$ for which the second law is violated $[. .$.$] goes to zero rapidly (exponentially) in the$ number of atoms and molecules in the system.' (Goldstein \& Lebowitz 2004, 57 $)^{8}$

This definition contains different elements that need to be distinguished for the discussion to follow. Let us begin by introducing some notation. $\Gamma_{M_{i}}^{(+)}$ is the subset of $\Gamma_{M_{i}}$ containing all those $x$ that lie on trajectories that come into $\Gamma_{M_{i}}$ from a macrostate of higher entropy and that leave $\Gamma_{M_{i}}$ entering into a macrostate of higher entropy; $\Gamma_{M_{i}}^{(+-)}, \Gamma_{M_{i}}^{(-+)}$and $\Gamma_{M_{i}}^{(--)}$are defined accordingly. These four subsets form a partition of $\Gamma_{M_{i}} \cdot{ }^{9}$ Furthermore, $\Gamma_{M_{i}}^{(+)}:=\Gamma_{M_{i}}^{(+)} \cup \Gamma_{M_{i}}^{(-)}$and $\Gamma_{M_{i}}^{(-)}:=\Gamma_{M_{i}}^{(+-)} \cup \Gamma_{M_{i}}^{(--)}$are the subsets of $\Gamma_{M_{i}}$ that have a higher and lower future entropy respectively.

The microstate $x \in \Gamma_{M_{i}}$ has the property 'being entropy increasing' (' $I$ ' for short) iff it lies on a trajectory that moves into a microstate of higher entropy when leaving $\Gamma_{M_{i}}$. Hence, $x$ has property $I$ iff $x \in \Gamma_{M_{i}}^{(+)}$. Entropy increasing states are typical in $\Gamma_{M_{i}}$ iff $\mu_{i}\left(\Gamma_{M_{i}}^{(+)}\right) \geq 1-\varepsilon$, where $\mu_{i}(\cdot):=$ $\mu(\cdot) / \mu\left(\Gamma_{M_{i}}\right)$ is the Lebesgue measure relative to $\Gamma_{M_{i}}$.

A system possesses the property of being 'globally entropy increasing' (' $G I$ ' for short) iff entropy increasing states are typical in every $\Gamma_{M_{i}}$ except the equilibrium macrostate itself (because, trivially, once the system has reached equilibrium entropy cannot further increase). Goldstein \& Lebowitz's explication of typicality (quoted above) amounts to saying that the system is GI. This can be seen as follows. In technical terms, Goldstein \& Lebowitz's condition is $\lim _{n \rightarrow \infty} \mu_{i}\left(\Gamma_{M_{i}}^{(-)}\right)=0$ for all $i$. Since the $\Gamma_{M_{i}}^{(+)}$, etc., form a partition of $\Gamma_{M_{i}}$, this is equivalent to $\lim _{n \rightarrow \infty} \mu_{i}\left(\Gamma_{M_{i}}^{(+)}\right)=1$ for all macrostates $M_{i}$ except the equilibrium macrostate. If we now assume (reasonably) that for $n \simeq 10^{23}$ we are already 'close' to the limit it follows that $\mu_{i}\left(\Gamma_{M_{i}}^{(+)}\right) \geq 1-\varepsilon$ for some small but finite $\varepsilon$.

${ }^{8}$ Explications of typicality very similar to this one can be found in Lebowitz (1993b, $7-8 ; 1999,348)$.

${ }^{9}$ I neglect the possibility that there maybe $x$ that come from or move into microstates of the same entropy. These cases could be accounted for by introducing the subsets $\Gamma_{M_{i}}^{(0+)}$, etc., and rephrasing the argument accordingly. One can easily see that this would not alter the conclusions that I reach and I therefore neglect them in the interest of ease of discussion and notion. 
We now face two questions. First, under what circumstances is it the case that a system is GI? Second, assuming we have a satisfactory answer to the first question, do we then have a good explanation for why the system approaches equilibrium? I discuss these questions in turn.

Goldstein \& Lebowitz offer the following answer to the question of when a system is $G I$ :

'Boltzmann then argued that given this disparity in sizes of different $M$ 's [i.e. the above-mentioned dominance of the equilibrium macrostate], the time evolved $\left[M_{x(t)}\right]$ will be such that $\left[\mu\left(M_{x(t)}\right)\right]$ and thus $\left[S_{B}(t)\right]$ will typically increase in accord with the law.' $(2004,57)$

They do not reference the work of Boltzmann they have in mind and so we have to work with their paraphrase of what they take to be Boltzmann's view. The argument seems to be that if it is the case that the ratio $\mu\left(\Gamma_{M_{e q}}\right) / \mu\left(\Gamma_{M_{i}}\right)$, where $M_{i}$ is a 'standard' non-equilibrium macrostate, is large (i.e. is of the magnitude of $10^{n}$ ), then the system is $G I$.

This is incorrect. Dominance of the equilibrium macrostate and being $G I$ are compatible with each other, but the latter does not follow from the former. From the fact that $\Gamma_{E}$ as a whole is almost entirely filled with equilibrium microstates and that therefore the measure of $\Gamma_{M_{e q}}$ is $10^{n}$ times the one of other macro-regions, it just does not follow that within every macro region $\Gamma_{M_{i}}^{(+)}$is typical. In fact, the dominance of the equilibrium macrostate is compatible, in principle, with it being the case that $\mu_{i}\left(\Gamma_{M_{i}}^{(+)}\right) \ll \mu_{i}\left(\Gamma_{M_{i}}^{(-)}\right)$for many low entropy macrostates $M_{i}$, in which case the system would fail to be GI. And the point is not one about there being the possibility of one or two macrostates behaving strangely and the system being 'a little bit non-GI'; it could be the case equilibrium microstates are typical with respect to $\Gamma_{E}$ as a whole, while entropy increasing behaviour is atypical in all low entropy macrostates.

That Account 3 fails is no surprise; whether or not a system is GI depends both on its dynamics and the construction of the macrostates and so it would be something of a miracle if one could prove systems to be $G I$ without even mentioning either of the two.

Given that we do not have a general argument for the claim that relevant systems are GI, the best we can do is look at examples. And here the evidence is mixed. One can show that the Kac ring model is GI (Lavis 2005, 259). 
However, GI seems to fail in other examples. Numerical considerations show that entropy increasing microstates are not typical within the low entropy macrostates of the baker's gas (as David Lavis pointed out to me in personal communication). So GI is not a trivial condition and there is a substantial question under which circumstances it holds.

There are also problems as regards the second question. To begin with, even if a system were GI it could still be the case that an approach to equilibrium would not take place. The problem is the following. Assume that the system is in macrostate $M_{i}$ at time $t_{1}$ and evolves into a macrostate $M_{j}$ of higher entropy at time $t_{2}$ (without passing through any other macrostates in-between). Furthermore assume that in both $M_{i}$ and $M_{j}$ entropy increasing microstates are typical. By construction, all states that evolve into $M_{j}$ from $M_{i}$ have to be either in $\Gamma_{M_{j}}^{(-+)}$or in $\Gamma_{M_{j}}^{(--)}$. In which one of these a particular $x \in M_{i}$ ends up is determined by the dynamics of the system, and it is possible that under certain dynamical laws most $x \in M_{i}$ end up moving into $\Gamma_{M_{j}}^{(-)}$. In this case most trajectories that are compatible with the system's actual past history move towards macrostate of lower entropy after $t_{2}$, despite the fact that $\Gamma_{M_{j}}^{(+)}$is typical in $\Gamma_{M_{j}}$.

So we need to add the further constraint that the dynamics of the system is such that for all (or at least most) contiguous macrostates $M_{i}$ and $M_{j}$, where $M_{i}$ has lower entropy than $M_{j}$, it be the case that the overwhelming majority of microstates in $\Gamma_{M_{i}}^{(+)}$move into $\Gamma_{M_{j}}^{(-+)}$. What condition could assure that this is the case? A possible answer to this question (or, rather, part of an answer) might be that the system has to show Goldilocks mixing (Earman 2006, 406). Although Earman discusses Goldilocks mixing in a different context and does not suggest that it is a solution to the current problem, it might at least be worth considering whether Goldilocks mixing, probably in conjunction with other conditions, proves useful in solving the problem at hand.

Furthermore there is the problem that most of the states that lie on trajectories that move towards higher entropy macrostates also have a high entropy past, i.e. behave un-thermodynamically. ${ }^{10}$ This can be seen as follows. By assumption $\Gamma_{M_{i}}^{(+)}$is typical, i.e. $\mu\left(\Gamma_{M_{i}}^{(+)}\right) \geq 1-\varepsilon$, and hence $\mu\left(\Gamma_{M_{i}}^{(-)}\right)<\varepsilon$. Since $\Gamma_{M_{i}}^{(-)}=\Gamma_{M_{i}}^{(--)} \cup \Gamma_{M_{i}}^{(+-)}$, we also have $\mu\left(\Gamma_{M_{i}}^{(+-)}\right)<\varepsilon$. The time reversal invari-

\footnotetext{
${ }^{10}$ A point to this effect was first made by Ehrenfest and Ehrenfest-Afanassjewa (1912, 32-34). However, their argument is based on an explicitly probabilistic model and so its relevance to deterministic dynamical system is tenuous.
} 
ance of the Hamiltonian dynamics implies $\mu\left(\Gamma_{M_{i}}^{(-+)}\right)=\mu\left(\Gamma_{M_{i}}^{(+-)}\right)$and therefore $\mu\left(\Gamma_{M_{i}}^{(-+)}\right)<\varepsilon$. With $\left.\Gamma_{M_{i}}^{(+)}\right)=\Gamma_{M_{i}}^{(-+)} \cup \Gamma_{M_{i}}^{(++)}$we obtain $\mu\left(\Gamma_{M_{i}}^{(++)}\right) \geq 1-2 \varepsilon$. Hence the typicality of $\Gamma_{M_{i}}^{(+)}$is hardly relevant to thermodynamic behaviour because the overwhelming majority of states in $\Gamma_{M_{i}}^{(+)}$do not exhibit the desired behaviour (i.e. they belong to $\Gamma_{M_{i}}^{(++)}$and hence have a high entropy past).

Remedy can be found in Albert (2000, Ch. 4), who suggests solving the problem by conditionalising on the past hypothesis (Albert does not put his argument in terms of typicality and uses probability language instead; what I am presenting here is an adaptation of his point to the present context). In technical terms that means that rather than pondering the question of whether microstates with high entropy future are typical with respect to the entire set $\Gamma_{M_{i}}$ we should require that this be the case with respect to $\Gamma_{M_{i}} \cap$ $\phi_{t}\left(\Gamma_{M_{p}}\right)$. The question now is whether states which evolve into macrostates of higher entropy are typical within that set.

And now we are back to the above problem, namely that this question cannot be answered without taking the dynamics of the system into account. There is nothing, in principle, to rule out that all states that satisfy this condition evolve into $\Gamma_{M_{j}}^{(-)}$once they leave $\Gamma_{M_{i}}$, in which case the system's entropy decreases once the states move from $\Gamma_{M_{j}}$ into the next macrostate. Albert $(2000,67,81-85,94-96)$ suggest ruling out that this happens by requiring that microstates that lead to un-thermodynamic behaviour are scattered in tiny clusters all over $\Gamma_{M_{i}}$. This is an interesting suggestion, but, again, there are neither a priori reasons nor plausibility arguments to suggest that this generally is the case in relevant systems. Whether or not this 'scattering condition' holds depends on the details of the dynamics and the construction of the macrostates, and merely asserting that the condition does hold is simply begging the question.

\section{Further Qualms}

There are five further problems for an approach to SM based on the notion of typicality: the justification of the Lebesgue measure as the relevant typicality measure, that the equilibrium macrostate may not be typical, that in interacting systems the largest macrostate may not be the equilibrium macrostate, the reliance on measures in general, and objections to the explanatory power of typicality even where it can be had. I will discuss each 
of these in turn.

First. Typicality judgements in all three accounts I have distinguished are made relative to the Lebesgue measure $\mu$. How can this be justified? Dürr (1998, Sec. 3) emphasises that the crucial criterion for the choice of a typicality measure is invariance over time. What is typical at some time $t$ also has to be typical at some earlier or later time $t^{\prime}$. In the context of SM this means that the typicality measure has to be invariant under the dynamics of the system (given by the flow $\phi_{t}$ ). As we have seen in the Section 2, the Lebesgue measure satisfies this criterion and therefore seems to be a natural choice.

Things are more involved, however. As Zanghì $(2005,189)$ points out, the Lebesgue measure $\mu$ may not be the only invariant measure in a particular system. For any specific Hamiltonian (equivalently for any specific $\phi_{t}$ ) there could also be invariant measures other than the Lebesgue measure whose explicit form depends on the details of the dynamics. Zanghì then points out that what makes the Lebesgue measure special is the fact that it is the only generical invariant measure, meaning that it is the only measure that is invariant under all Hamiltonian flows.

It is not clear, however, that this fact is relevant for the problem at hand. Each system is governed by one, and only one, Hamiltonian and it is therefore not clear why the fact that the Lebesgue measure is the only measure that is invariant under all Hamiltonians is relevant for typicality judgements in this system. If it happens that there is a measure $\mu^{\prime}$ which is invariant under the dynamics of the system under investigation and which is non-equivalent to the Lebesgue measure, why should we not make typicality judgments about this system with respect to $\mu^{\prime}$ ? This question is particularly pressing for those - like most Boltzmannians - who take the relevant system to be the universe as a whole and the past state the Big Bang. There is only one universe and there is only one Hamiltonian flow in this universe. What reason could there be to prefer $\mu$ to $\mu^{\prime}$ to make typicality judgements in this universe?

There is no obvious answer to this question. But maybe none is needed. A similar issue arises in the case of the Galton board. Maudlin (2007) points out that atypical initial conditions in that have measure zero and hence typicality judgments remain unaltered under a change of measures as long as the alternative measure $\mu^{\prime}$ is absolutely continuous with the Lebesgue measure $\mu .{ }^{11}$ So there is actually no need to worry about the question of

\footnotetext{
${ }^{11} \mathrm{~A}$ measure $\mu^{\prime}$ is absolutely continuous with $\mu$ iff for any measurable region $A \subseteq \Gamma_{E}$ :
} 
picking the 'right' measure because under all choices the same sets come out as typical, which is all we need.

It is not clear whether this strategy is available in SM. First, Maudlin's argument only applies to measures that are absolutely continuous with the Lebesgue measure. So we would need an argument for the conclusion that all invariant measures have this property. This may or may not be the case; at any rate it is not a priori clear that this is so. ${ }^{12}$ Second, one would have to show that it is indeed the case that all atypical sets have Lebesgue measure zero. Again, this is not evidently so. Even in a simple system like the Galton Board a host of drastic idealisations are needed to reach this conclusion (for instance, one has to assume that the board is infinitely long and that all the nails are perfectly symmetrical), which then still is only supported by a plausibility argument and not a rigorous proof. It is not clear that idealisations of this sort can be made of our universe, and even if they can this may not yield the desired result because the dynamics of our universe is much more complex than the one of the Galton Board and hence it is at least a possibility that some sets of finite measure are atypical. If this is the case and if there is an invariant measure $\mu^{\prime}$ (which could even be absolutely continuous with $\mu$ ), it might be the case that $\mu^{\prime}$ assigns high weights to sets that come out small under $\mu$, which would reverse typicality judgements. Hence what is typical with respect to $\mu$ would come out to be atypical with respect to $\mu^{\prime}$ and vice versa. There is no a priori reason to rule out this possibility.

Second. A further difficulty concerns the dominance of the equilibrium macrostate. As I have briefly mentioned above, from the fact that the equilibrium macrostate is larger than any other macrostate one cannot infer that it is typical. Lavis $(2005,255-258)$ points out that entropy levels can be degenerate, meaning that there may be more than one macrostate for which the Boltzmann entropy assumes a particular value. More precisely, consider a particular macrostate $M_{j}$, construct the set $\left\{M_{i} \mid S_{B}\left(M_{i}\right)=S_{B}\left(M_{j}\right), i=\right.$ $1, \ldots, m\}$ of all macrostates that have the same entropy as $M_{j}$, and let $\omega_{j}$ be the number of macrostates in this set; $\omega_{j}$ is the degeneracy of the en-

if $\mu(A)=0$ then $\mu^{\prime}(A)=0$. More colloquially, a measure $\mu^{\prime}$ is absolutely continuous with another measure $\mu$ if it assigns measure zero to all sets that are assigned measure zero by $\mu$, while, possibly, assigning different values to the sets to which $\mu$ assigns non-zero measure.

${ }^{12}$ Maybe an defence along the lines of Malement and Zabell (1980) would fit the bill, but this would need to ba argued in detail. 
tropy value $S_{B}\left(M_{j}\right)$. The important point is that these degeneracies may be large enough for it to be the case that the non-equilibrium macrostates associated with a particular entropy value together take up a larger chunk of the phase space than the equilibrium macrostate; that is, it may be the case that $\omega_{j} \mu\left(\Gamma_{M_{j}}\right)>\mu\left(\Gamma_{M_{e q}}\right)$, for some non-equilibrium macrostate $M_{j}$. Lavis shows that this is not only a theoretical possibility. He points out that it is exactly what happens in the case of the baker's gas (ibid.) and in the Kac ring model (Lavis 2008, Sec. 2), in which the proportion of the phase space occupied by the maximum entropy state even decreases as $n$ becomes large. Of course, real systems are neither baker's gases nor Kac rings and so this problem with degeneracies may not surface in more 'realistic' systems. However, whether or not this is the case depends on the details of the system and one would have to show that in the systems of interest no such degeneracies crop up.

Third. So far we have assumed that the equilibrium macrostate is the largest of all macrostates (and the second problem concerns the question of whether this state is typical in $\Gamma_{E}$ ). Although this is usually stated as if it were a general truism, it is proven only for an ideal gas, i.e. a system of noninteracting particles. In broad outline, the reasoning, invented by Boltzmann in 1877 and now usually referred to as the 'combinatorial argument', is as follows (for an in-depth discussion see Uffink (2007, 974-983). Consider the phase-space of one gas molecule; the state of the entire gas (consisting of $n$ molecules) is specified by $n$ labeled points in this space. Now put a grid-like partition on it with the border of the cells running in the directions of the momentum and position axes. Every one of the $n$ points comes to lie within a particular cell of the partition. A specification of which point lies in which cell is called an 'arrangement'; a specification of how many points (no matter which ones) are in each cell is a 'distribution'. Boltzmann then considered how many arrangements are compatible with each distribution and associated the logarithm of this number, $W$, with the entropy of the system (this can be shown to be equivalent to the definition of the Boltzmann entropy given in Section 2). One can then prove that $W$ is proportional to the Lebesgue measure of the region of the $n$-particle phase space, $\Gamma_{E}$, corresponding to the distribution. By construction it follows that largest macrostate is associated with the largest Boltzmann entropy, and this macrostate is then considered the equilibrium macrostate.

However, we should not be mislead by the suggestive use of the word 'entropy'; the argument so far is just a combinatorial exercise and its physical 
relevance yet needs to be shown. And this is where the crucial assumptions enter. Suppose that the energy of a molecule only depends on the cell in which it is (but not on where all the other molecules are) and that the total energy of the system is the sum of these 'individual' energies. Under this assumption (and the further assumption that the number of molecules in each cell is far greater than one) one can prove that the velocity distribution of those phase points that are in the maximum Boltzmann entropy region is the Maxwell-Boltzmann equilibrium distribution. For this reason it is indeed legitimate to associate equilibrium with maximum Boltzmann entropy.

The crucial assumption in this proof is that the entropy of a molecule only depends on the cell in which it is, as this amounts to nothing less than the assumption that there is no interaction between the molecules; in other words, it amounts to assuming that the system is an ideal gas (Uffink 2007, 976). Hence, for systems that are not ideal gases there is at least a question of whether their equilibrium macrostate can be associated with the largest macrostate. And this is more than an academic point. Most systems, not least the universe as a whole, are not ideal gases, not even approximately, and it is not clear whether in such systems the equilibrium macrostate can legitimately be associated with the largest macrostate (i.e. the one for which the Boltzmann entropy is maximal).

In fact, it is a real option that this is not the case. Consider a system of gravitating particles. These particles attract each other and hence have the tendency to clump together. So if it happens that a large amount of these are distributed evenly over a bounded space, then they will move together and eventually form a lump. However, the volume corresponding to a lump is much smaller than the one corresponding to the original spread out state, and hence it seems that the system evolves from a high to a low entropy state. This conclusion is usually blocked by pointing out that the loss in volume in configuration space is compensated by a corresponding increase in volume in momentum space, and as a result entropy does not decrease after all. But whether this is true depends on the details of the system at hand. There are situations in which this is not the case, for instance one in which all particles end up moving around with almost the same velocity and hence occupy only a small volume of momentum space. So one would need to argue that the systems of interest are not of this kind. ${ }^{13}$

\footnotetext{
${ }^{13}$ See Callender (2008) for a further discussion of the problems that arise in connection with gravity.
} 
Fourth. One of the main objections against approaches to SM that invoke ergodicity is the so-called 'measure zero problem' (see van Lith (2001) for a discussion). The results of ergodic theory come with the qualification 'almost everywhere' - i.e. everywhere except, perhaps, for a set of measure zero which is commonly understood as suggesting that sets of measure zero can be ignored because they are somehow 'sparse'. This piece of common wisdom has been criticised as untenable. Sets of measure zero need not be 'small' at all (e.g. the rational numbers have measure zero within the real numbers and yet there are 'many' of them) and, as Sklar (1993, 182-188) points out, a set of measure zero need not be (or even appear to be) negligible if sets are compared with respect to properties other than their measures. For instance, we can judge the 'size' of a set by its cardinality or Bair category rather than by its measure which may lead to different conclusions about a set's 'size'.

This point has to do with the use of measures in general and is not specific to ergodic theory. In fact, because typicality is determined with respect to a measure, approaches to SM appealing to typical behaviour face a very similar problem: sets of measure zero (like the rational numbers) are classified as atypical and it is suggested that these can therefore be neglected. However, echoing Sklar's point, sets that come out as atypical when compared to other sets with respect to their measures may not come out as atypical when compared with respect to some other property (such as their Bair category). So we face the question of what conveys upon measures a privileged status when it comes to judging typicality.

Fifth. The basic strategy of typicality-based approaches is to explain $X$ by pointing out that $X$ is typical. For instance, when asked why a system approaches equilibrium the proponent of Approach 2 answers that this is because initial conditions that lie on trajectories that approach equilibrium are typical in the set of all initial conditions. It is questionable whether this answer is satisfactory, even if the desired behaviour in fact turns out to be typical. The problem is, again, parallel to one that threatens the ergodic approach. As Sklar (1973, 210-211) points out in his critique of this approach, from the fact that an initial condition lies within a set of measure zero we cannot infer that that this initial condition does not occur. Whether the system has a particular initial condition is a factual question, and as such it has to be settled by an appeal to matters of fact and not measures of sets; to explain why the system exhibits entropy increasing behaviour we need an argument for the conclusion that the system indeed started out in a typical initial condition, but that these are of measure (close to) one does not give 
us such an argument.

But now the significance of typicality seems to have evaporated entirely. All we need to explain a system's actual behaviour is its actual initial condition is one which, under the dynamical law governing the system's evolution, evolves in a thermodynamic way. Whether or not this initial condition is also typical is simply irrelevant. So typicality does not play a role in explaining the behaviour of a particular system (like, for instance, our universe).

One could reply that the notion of explanation that underlies this criticism is too metaphysical (in that it implicitly assumes that an explanation of $X$ has to show that $X$ must happen under the given circumstances) and that a different, less assuming, notion should be applied. An obvious candidate is rational expectability. On this notion conception of explanation we explain $X$ by showing that it is rationally expectable that $X$ occurs. This seems to square well both with typicality, because if a behaviour is typical we are surely rationally justified in believing that it occurs most of the time. This also squares well with the intuition driving the (probabilistic version of) the covering law account of scientific explanation, according to which we explain $X$ if we can show that $X$ is very likely to occur.

But even if we are willing to set all the well-known problems of accounts of this sort aside (see Salmon (1992) for survey), such an account would not sit well with the general hostility towards epistemic approaches that permeates this literature, in particular the flamboyant rejection of an epistemic interpretation of probability (see for instance Albert (2000, 64), Loewer (2001, 611), and Goldstein $(2001,48)$. But if we reject an epistemic notion of explanation, it remains unclear how we can explain the behaviour of a particular system (this universe) by appeal to typicality.

\section{Conclusion}

I have distinguished three different ways in which typicality is used to explain why systems approach equilibrium and argued that none of them is successful. The first is false for mathematical reasons, while the latter two prima facie provide a restatements of the problem rather than a solution because they do not provide dynamical conditions. But even if these difficulties can be solved, there are further conceptual problems. First, all accounts attribute a special status to the Lebesgue measure, but the justifications of this choice do not seem to be conclusive. Second, it is not clear whether 
the equilibrium macrostate is typical in $\Gamma_{E}$. Third, typicality arguments are usually put forward in the context of ideal gases, and there are serious questions about whether they can be carried to gravitating systems. Fourth, like approaches based on ergodicity, typicality arguments dismiss sets of measure zero as 'negligible'. It is not clear, however, how this can be justified. Finally, it is questionable whether an appeal to what typically happens has any explanatory force at all when it comes to explaining what happens in a particular system.

\section{Acknowledgements}

Special thanks goes to David Lavis for many illuminating discussions on SM in general, and the Boltzmannian approach in particular. I also would like to thank Craig Callender, Stephan Hartmann, Carl Hoefer, Wolfgang Pietsch, Charlotte Werndl, and two anonymous referees for valuable comments on earlier drafts. Thanks to Jean Bricmont for a helpful email conversation on his mixing condition discussed in Section 3, and to Detlef Dürr for drawing my attention to omissions in my first bibliography. Many thanks to Flavia Padovani for helping me with those passages in Zanghì's chapter that were beyond the reach of my 'FAPP Italian'. Thanks to Mauricio Suárez for organising the workshop at which this paper has first been presented, and thanks to the audiences in Madrid and Oxford for stimulating discussions. Finally, I would like to acknowledge financial support from two project grants of the Spanish Ministry of Science and Education (SB2005-0167 and HUM2005-04369).

\section{Bibliography}

Albert (2000): Time and Chance. Cambridge/MA and London: Harvard University Press.

Arnold, Vladimir I. (2006): Ordinary Differential Equations. Berlin and Heidelberg: Springer.

Boltzmann, Ludwig (1877): 'Über die Beziehung zwischen dem zweiten Hauptsatze der mechanischen Wärmetheorie und der Wahrscheinlichkeitsrechnung resp. den Sätzen über das Wärmegleichgewicht. Wiener Berichte 
76, 373-435. Reprinted in F. Hasenöhrl (ed.): Wissenschaftliche Abhandlungen. Leipzig: J. A. Barth 1909, Vol. 2, 164-223.

Bricmont, Jean (1996). 'Science of Chaos or Chaos in Science?', in P. R. Gross, N. Levitt, \& M. W. Lewis (eds.): The flight from science and reason, Annals of the New York Academy of Sciences, Volume 775, 131175 .

- (2001): 'Bayes, Boltzmann and Bohm: Probabilities in Physics', in Bricmont et al. 2001, 3-21.

-, Detlef Dürr, Maria Carla Galavotti, Gian Carlo Ghirardi, Francesco Petruccione and Nino Zanghì (eds.) (2001): Chance in Physics: Foundations and Perspectives. Berlin and New York: Sringer.

Callender, Craig (1999): 'Reducing Thermodynamics to Statistical Mechanics: The Case of Entropy', Journal of Philosophy 96, 348-373.

- (2001): 'Taking Thermodynamics Too Seriously', Studies in the History and Philosophy of Modern Physics 32, 539-53.

- (2008): 'The Past Hypothesis Meets Gravity', forthcoming in Gerhard Ernst and Andreas Httemann (eds.): Time, Chance and Reduction. Philosophical Aspects of Statistical Mechanics. Cambridge: Cambridge University Press.

Dürr, Detlef (1998): 'Über den Zufall in der Physik', manuscript presented at the 1998 Leopoldina Meeting in Halle. Available at http://www.mathematik.unimuenchen.de/\%7Eduerr/Zufall/zufall.html

— (2001): Bohmsche Mechanik als Grundlage der Quantenmechanik. Berlin: Springer.

- (2005): 'Chance in Physics', unpublished lecture notes of the two lectures given at the 4th International Summer School, University of Konstanz, Germany.

-, Sheldon Goldsein and Nino Zanghì (1992): 'Quantum Equilibrium and the Origin of Absolute Uncertainty', Journal of Statistical Physics 67, 843-907. 
Earman, John (2006): 'The "Past Hypothesis": Not even False', Studies in History and Philosophy of Modern Physics 37, 399-430.

— and Miklós Rédei (1996): 'Why Ergodic Theory Does Not Explain the Success of Equilibrium Statistical Mechanics', British Journal for the Philosophy of Science 47, 63-78.

Ehrenfest, Paul and Ehrenfest-Afanassjewa, Tatiana (1912/1959): The Conceptual Foundations of the Statistical Approach in Mechanics. Mineola/New York: Dover 2002. (First published in German in 1912; first English Translation 1959.)

Frigg, Roman (2008): 'A Field Guide to Recent Work on the Foundations of Statistical Mechanics', in Dean Rickles (ed.): The Ashgate Companion to Contemporary Philosophy of Physics. London: Ashgate, 99-196.

Galvan, Bruno (2006): 'Typicality vs. Probability in Trajectory-Based Formulations of Quantum Mechanics', Foundations of Physics 37, 15401562 .

Goldstein, Sheldon (2001): 'Boltzmann's Approach to Statistical Mechanics', in: Bricmont et al. 2001, 39-54.

- and Joel L. Lebowitz (2004): 'On the (Boltzmann) Entropy of NonEquilibrium Systems', Physica D 193, 53-66.

— Joel. L. Lebowitz, Roderich Tomulka and Nino Zanghì (2006): 'Canonical Typicality', Physical Review Letters 96, Issue 5.

Hitchcock, Christopher (ed.): Contemporary Debates in Philosophy of Science. Oxford and Malden/MA: Blackwell.

Lavis, David (2005): 'Boltzmann and Gibbs: An Attempted Reconciliation', Studies in History and Philosophy of Modern Physics 36, 245-273.

- (2008): 'Boltzmann, Gibbs and the concept of Equilibrium', forthcoming in Philosophy of Science (Proceedings).

Lebowitz, Joel L. (1993a): 'Boltzmann's Entropy and Time's Arrow', Physics Today, September Issue, 32-38. 
- (1993b): 'Macroscopic Laws, Microscopic Dynamics, Time's Arrow and Boltzmann's Entropy', Physica A 194, 1-27.

- (1999): 'Statistical Mechanics: A Selective Review of Two Central Issues', Reviews of Modern Physics 71, 346-357.

Malament, David B. and Sandy L. Zabell (1980): 'Why Gibbs Phase Averages Work', Philosophy of Science 47, 339-349.

Maudlin, Tim (2007): 'What Could Be Objective About Probabilities?', Studies in History and Philosophy of Modern Physics 38, 275-291.

Penrose, Roger (1989): The Emperor's New Mind. Oxford: Oxford University Press.

Salmon, Wesley (1992): 'Scientific Explanation', in Marrilee Salmon et al. (1992): Introduction to the Philosophy of Science. Indianapolis and Cambridge: Hackett, 7-23.

Sklar, Lawrence (1973): 'Statistical Explanation and Ergodic Theory', Philosophy of Science 40, 194-212.

- (1993): Physics and Chance. Philosophical Issues in the Foundations of Statistical Mechanics. Cambridge: Cambridge University Press.

Uffink, Jos (2007): 'Compendium of the Foundations of Classical Statistical Physics', in: Jeremy Butterfield and John Earman (eds.): Philosophy of Physics. Amsterdam: North Holland, 923-1047.

van Lith, Janneke (2001): 'Ergodic Theory, Interpretations of Probability and the Foundations of Statistical Mechanics', Studies in History and Philosophy of Modern Physics 32, 581-594.

Volchan, Sérgio B. (2007): 'Probability as Typicality', Studies in History and Philosophy of Modern Physics 38, 801-814.

Zanghì, Nino (2005): 'I Fondamenti Concettuali dell'Approccio Statistico in Fisica', in Valia Allori, Mauro Dorato, Federico Laudisa and Nino Zanghì: La Natura Delle Cose. Introduzione ai Fundamenti e alla Filosofia della Fisica. Roma: Carocci. 